\title{
Integrated traditional Chinese medicine improves acute pancreatitis via the downregulation of PRSS1 and SPINK1
}

\author{
QIANG GAO $^{1,2}$ and NUSHENG LIANG ${ }^{3}$ \\ ${ }^{1}$ Physician Department, Health Service Center, Nyalam, Tibet 858300; ${ }^{2}$ Gastroenterology Department, \\ Yantai Hospital of Traditional Chinese Medicine, Yantai, Shandong 264002; ${ }^{3}$ Department of \\ Gastroenterology, First Hospital of Tianjin, Tianjin 300193, P.R. China
}

Received June 8, 2014; Accepted December 19, 2014

DOI: $10.3892 / \mathrm{etm} .2015 .2191$

\begin{abstract}
Integrated traditional Chinese medicine (ITCM) is known to improve health in patients with acute pancreatitis (AP); however, the molecular mechanisms underlying this effect are unknown. AP is associated with the expression of PRSS1 and SPINK1. Thus, the present study aimed to investigate whether ITCM was able to ameliorate AP by regulating the expression levels of protein, serine 1 (PRSS1) and serine peptidase inhibitor, Kazal type 1 (SPINK1). A total of 100 AP patients were divided at random into two groups. The treatment group were treated externally with a herbal ITCM preparation, while the control group received a routine placebo treatment. The mRNA and protein expression levels of PRSS1 and SPINK1 were subsequently compared between the two groups. The results revealed that the health of the patients who had received ITCM improved significantly when compared with the control group patients $(\mathrm{P}<0.05)$. In addition, the expression levels of PRSS1 and SPINK1 were found to be lower in the treatment group when compared with the control group $(\mathrm{P}<0.05)$. Therefore, ITCM exhibited a significant therapeutic effect on AP and produced no side effects since the treatment was applied externally. ITCM may ameliorate AP by downregulating the expression of PRSS1 and SPINK1; thus, should be considered as a potential therapy for the development of drugs against AP.
\end{abstract}

\section{Introduction}

Acute pancreatitis (AP) is a common disease of the digestive system, characterized by acute, severe symptoms, a variety of complications and a high rate of mortality $(1,2)$. Paralytic intestinal obstruction is an early clinical symptom of AP,

Correspondence to: Dr Qiang Gao, Physician Department, Health Service Center, Room 306, Building 25, Chongdui Road, Nyalam, Tibet 858300, P.R. China

E-mail: gaoqiangtib@163.com

Key words: integrated traditional Chinese medicine, acute pancreatitis, protein, serine 1 , serine peptidase inhibitor, Kazal type 1 occurring in $>80 \%$ of cases (3). The pathogenesis of pancreatitis is considered to be associated with the inflammatory response and an abnormal immune reaction (4,5), which are caused by the activation of pancreatic enzymes, fermentemia and numerous cytokines and inflammatory mediators (6). The intestinal hypomotility caused by the pathogenesis of pancreatitis may therefore lead to AP (7). In the course of AP, the intestinal mucosa barrier is damaged by the translocation of intestinal bacteria and endotoxins (8-10). AP aggravates the inflammatory reaction, interferes with the nerve function of the gastrointestinal tract, compromises blood circulation in the intestines and damages the intestinal mucosa. This damage may result in an intestinal motility disorder, flatulence, bowel dilatation and abdominal pressure, which may in turn affect the gastrointestinal blood supply and increase microcirculation $(11,12)$. In addition, the large volume of liquid exudation, damage to the intestinal mucosal barrier, bacterial translocation and the absorption of toxins may aggravate shock and systemic infection, resulting in mortality $(13,14)$. There is a close association between AP and infective pancreatic necrosis $(15,16)$.

However, the etiology of AP is complicated and the appropriate therapy for the disease remains contested $(2,17)$. The application of acupuncture, when combined with Western medicine, has been considered an effective therapy for AP (18). The majority of treatments for AP have evident side effects (19), which limit their use in the treatment of AP. Integrated traditional Chinese medicine (ITCM) has been practiced for over 1,000 years and is widely used as an external treatment for AP, with few side effects (20-22). The present study reports a novel ITCM treatment that is an effective therapy for AP. However, the mechanism underlying the effect of ITCM in the treatment of AP remains unknown. The protein, serine 1 (PRSS1) gene, which expresses cationic trypsinogen, is associated with hereditary pancreatitis and characterized by acute inflammation of the pancreas (23). SPINK1, which codes for serine protease inhibitor Kazal-type 1, is an additional gene regarded as a disease modifier of AP $(24,25)$. Thus, AP has been shown to be associated with the expression of PRSS1 and serine peptidase inhibitor, Kazal type 1 (SPINK1) $(24,26,27)$. The aim of the present study was to investigate whether ITCM may ameliorate AP by regulating the expression of PRSS1 and SPINK1. 


\section{Materials and methods}

Participants. The study protocols on human subjects and consent documents were approved by the Human Research Ethics Committee of Yantai Hospital of Traditional Chinese Medicine (Yantai, China). In addition, written consent documents were obtained and the data were collected with a Certificate of Confidentiality protecting patient privacy. A total of 100 AP patients were recruited from the Yantai Hospital of Traditional Chinese Medicine between March 2009 and March 2014 in order to rule our or prevent any genetic differences. All patients were unrelated, ethnic Han Chinese with similar cultural and economic backgrounds.

AP patients were divided at random into two groups (Table I). In total, 100 patients (age range, 40-55 years) were examined who were reported to be suffering with AP, but had no symptoms of other intestinal or inflammatory diseases. All eligible cases had their diagnoses confirmed by histological examination. The course of the disease ranged between $3 \mathrm{~h}$ and three days. Of the 100 cases, 48 were biliary in origin, 40 cases were due to overeating and overdrinking and 12 cases were the result of unidentified causes. In 94 cases, the patient was suffering from AP for the first time, while the remaining six cases were recurrent.

Diagnostic criteria and disease classification. The diagnostic criteria for AP patients were in accordance with a previous study (28), and were based upon the elevated levels of pancreatic enzymes in the blood and urine. According to the diagnostic criteria, patients presenting with the following symptoms were considered to suffer from AP: Upper abdominal pain; enhanced levels of lipase, examined using a Human Pancreatic Lipase ELISA kit (Wuhan Jiacheng Biotechnology Co., Ltd., Wuhan, China); ultrasonography (US) scans (GE LOCIQ 400; GE Healthcare, Milwaukee, WI, USA) demonstrating dilation of the common bile duct or swelling of the pancreas. Clinical manifestations of AP included sudden and sharp cutting pains in upper abdomen, nausea and vomiting, fever, several days without defecation, abdominal distension, dry and bitter taste in the mouth, coated and dry or burned black tongue and forceful sphygmus. All other abdominal diseases associated with pancreatitis were ruled out. In addition, peripheral blood tests revealed increases in the number of peripheral white blood cells, neutrophils and serum amylase, while an increase in the level of urine amylase was also observed. Diffuse enlargement of the pancreas was observed in B-mode US and computed tomography (CT) examinations. The bioactivities of serum and urine amylase were analyzed in accordance with procedures described in previous studies $(29,30)$. Blood lipase levels were also measured, since lipase is a more sensitive and specific indicator of AP compared with other pancreatic enzymes. CT scanning is the preferred method for confirming the image findings of AP.

AP can be classified as mild, moderate or severe, according to the disease severity (31). Mild AP patients exhibit no organ failure, nor any local or systemic complications. Moderate AP is characterized by the presence of transient organ failure, while severe AP patients exhibit persistent organ failure for $>48 \mathrm{~h}$.

Therapeutic methods. Experimental group patients were externally administered with an ITCM preparation. The
ITCM preparation was composed and prepared in accordance with previous studies $(32,33)$ as follows: $30 \mathrm{~g}$ Chinese rhubarb, $15 \mathrm{~g}$ Citrus aurantium, $15 \mathrm{~g}$ magnolia bark, $10 \mathrm{~g}$ mirabilite, $10 \mathrm{~g}$ Pinellia ternata, $10 \mathrm{~g}$ Coptis chinensis, $15 \mathrm{~g}$ Scutellaria baicalensis, $10 \mathrm{~g}$ Gardenia, 15 g Radix Paeoniae Alba, $15 \mathrm{~g}$ tree peony bark, $15 \mathrm{~g}$ Radix Paeoniae Rubra, $15 \mathrm{~g}$ Rhizoma Corydalis, $10 \mathrm{~g}$ Bupleurum and $10 \mathrm{~g}$ licorice. The materials were ground to a powder using a high-pressure homogenizer (Jiangsu Makwell Machinery Co., Ltd., Huai'an, China). These powders were dissolved in $500 \mathrm{ml}$ water with $200 \mathrm{~g}$ starch, and the mixture was concentrated and dried to form pastes of $\sim 5 \times 5 \times 1 \mathrm{~cm}$. The pastes were applied to the acupuncture points $(18,34)$, Yishu, Zhongwan, Tianshu, Zusanli, Guan Yuan and Pishu, twice daily. The dose of rhubarb and mirabilite was adjusted according to the severity of the patients' abdominal distention and pain. In addition, the composition of the powder was modified according to patient symptoms, tongue coating and pulse. The control group patients received acupuncture point application with a placebo treatment, containing a mixture of cornmeal, starch and flour.

Curative standard. A curative standard was defined in order to categorize the efficacy of the ITCM treatment. The standard was defined as the regression of symptoms, including abdominal pain and distension, fever, vomiting and passing of feces or wind. Furthermore, improvements to the patient abdominal muscle tension, abdominal tenderness, rebound tenderness and bowel sound were considered to indicate successful therapy. In addition, blood and urine amylase levels, and routine blood and liver function tests, were returned to normal, and the extent of pancreatic edema had improved when compared with the previous abdominal B-mode US and CT examinations. The following categories were used for defining the therapeutic results: Excellent, reaching the aforementioned standard in four days; effective, reaching the aforementioned standard in seven days; inefficient, failing to reach the standard after eight days.

Sample isolation. Sinker-assisted endoscopic submucosal dissection (ESD) is an effective technique for the removal of minus superficial pancreatic tissues (35); thus, this technique is the preferred approach for obtaining surgical specimens from AP patients. Ethical issues were considered to be very important. It was a priority to ensure that patient participation was entirely voluntary and that patient privacy was maintained. Experts conducting the study were not to attempt to persuade participants to donate any tissues if the participant was at all in doubt regarding the safety or ethics of the study.

Potential mediating factors. A number of confounding factors have been reported to be associated with an increased risk of AP (36-40). A previous meta-analysis indicated that obesity was associated with a higher risk of AP (41); therefore, the body mass index (BMI) of the patients was measured in the present study. Gender has also been regarded as an important determinant of outcome in AP patients (42); thus, the data for the relative female and male reactions to AP treatment were also surveyed. Alcohol consumption can increase the risk of AP $(43,44)$; therefore, all the subjects were non-drinkers, having never consumed alcohol, in order to avoid interference. To avoid age-related issues $(45,46)$, all individuals were 
Table I. Patient characteristics prior to ITCM treatment.

A, Male patients

\begin{tabular}{lccr}
\hline Characteristics & Experiment group & Control group & P-value \\
\hline Cases (n) & 32 & 32 & $>0.05^{\mathrm{a}}$ \\
Age (years) & $50.5 \pm 4.9$ & $50.8 \pm 4.8$ & $>0.05^{\mathrm{b}}$ \\
BMI $\left(\mathrm{kg} / \mathrm{m}^{2}\right)$ & $25.7 \pm 6.1$ & $26.0 \pm 6.7$ & $>0.05^{\mathrm{b}}$ \\
Daily calorie intake (kcal) & $2255 \pm 377$ & $2188 \pm 409$ & $>0.05^{\mathrm{b}}$ \\
AP classification (n) & & 17 & $>0.05^{\mathrm{a}}$ \\
Mild & 18 & 8 & $>0.05^{\mathrm{a}}$ \\
Moderate & 8 & 7 & $>0.05^{\mathrm{a}}$ \\
Severe & 6 & & \\
\hline
\end{tabular}

B, Female patients

\begin{tabular}{lccr}
\hline Characteristics & Experiment group & Control group & P-value \\
\hline Cases (n) & 18 & 18 & $>0.05^{\mathrm{a}}$ \\
Age (years) & $46.9 \pm 6.7$ & $48.1 \pm 6.9$ & $>0.05^{\mathrm{b}}$ \\
BMI $\left(\mathrm{kg} / \mathrm{m}^{2}\right)$ & $25.1 \pm 5.8$ & $25.7 \pm 5.5$ & $>0.05^{\mathrm{b}}$ \\
Daily calorie intake (kcal) & $2033 \pm 249$ & $2099 \pm 276$ & $>0.05^{\mathrm{b}}$ \\
AP classification (n) & & 8 & $>0.05^{\mathrm{a}}$ \\
Mild & 8 & 5 & $>0.05^{\mathrm{a}}$ \\
Moderate & 5 & 5 & $>0.05^{\mathrm{a}}$ \\
Severe & 5 & &
\end{tabular}

P-value calculated with the ${ }^{\mathrm{a}} \mathrm{t}$-test and ${ }^{\mathrm{b}} \chi^{2}$ test. ITCM, integrated traditional Chinese medicine; BMI, body mass index; AP, acute pancreatitis.

aged between 40 and 55 years-old. All volunteers reported a food consumption frequency of three meals per day, and the food consumption was consistent to a healthy Nordic food index $(47,48)$. Calorie intake was calculated according to self-reported assessment of daily calorie intake (49).

ELISA. The concentration of lipase was examined using an ELISA, with lipase antibodies purchased from Wuhan Jiacheng Biotechnology Co., Ltd. (Wuhan, China). Biopsy specimens were ground using liquid nitrogen. The grounded specimens were diluted by $1 / 200$ in phosphate-buffered saline (PBS) and transferred to antibody-coated wells. Following the manufacturer's instructions, all wells were washed three times with PBS, and IgG (Immuno Pure ${ }^{\circledR}$ peroxidase-conjugated goat anti-human IgG; 21348; Beijing Jiamay Biotechnology Ltd., Beijing, China) was added and incubated for $30 \mathrm{~min}$. After washing, 3,3',5,5'-tetramethylbenzidine (54827-17-7; Robiot Co., Ltd., Nanjing, China) was added and cultured for $10 \mathrm{~min}$ for visualization. Finally, the absorbance of the mixture was examined at $450 \mathrm{~nm}$ using a SM600 ELISA plate reader (Shanghai Utrao Medical Instrument Co., Ltd., Shanghai, China). The concentration of lipase was calculated according to a standard calibrator curve.

Quantitative reverse transcription polymerase chain reaction ( $q R T-P C R)$. The mRNA expression levels of PRSS1 and
SPINK1 were assessed using qRT-PCR. RNA was extracted from the AP tissues using a GenElute ${ }^{\mathrm{TM}}$ Mammalian Total RNA Miniprep kit (RTN10; Sigma-Aldrich Trading Co., Ltd., Shanghai, China). Initial cDNA was amplified from RNA using a random primer. The cDNA molecules were used as a template for qRT-PCR of the PRSS1 and SPINK1 transcripts on a rapid qTOWER 2.0 (Analytik Jena AG, Thuringia, Germany). The primers for qRT-PCR were as follows: PRSS1 (GenBank no. BC103998.2) sense, 5'-AGGGGAATGAGCAGTTCATC-3' and antisense, 5'-CACCAGAACTCAGAGTGTTG-3'; SPINK1 (GenBank no. NM003122.3), sense, 5'-GAAGAGACGTGG TAAGTGCG-3' and antisense, 5'-CCATCAGTCCCACAG ACAGGG-3'; GAPDH sense, 5'-CCCTTCATTGACCTCAAC TAC-3' and antisense, 5'-CCACCTTCTTGATGTCATCAT-3'. GAPDH was used as an internal control. All genes were amplified by 30 cycles of heating for $30 \mathrm{sec}$ at $94^{\circ} \mathrm{C}$, followed by $1 \mathrm{~min}$ at $60^{\circ} \mathrm{C}$. The quality of the synthesized cDNA was determined using GAPDH as the reference gene.

Protein expression levels of PRSS1 and SPINK1. Protein expression levels of PRSS1 and SPINK1 were determined by western blot analysis. The protein was isolated from selected AP tissues and separated using 12\% SDS-PAGE. The separated protein was electrotransferred onto polyvinylidene fluoride membranes (Bio-Rad Laboratories, Inc., Hercules, CA, USA), which were blocked with $5 \%$ non-fat dry milk 
Table II. Comparison of clinical characteristics for AP patients prior to and following ITCM treatment.

\begin{tabular}{|c|c|c|c|c|c|c|}
\hline \multirow[b]{2}{*}{ Clinical characteristic } & \multicolumn{3}{|c|}{ Before treatment } & \multicolumn{3}{|c|}{ After treatment } \\
\hline & $\begin{array}{l}\text { Experiment } \\
\text { group }\end{array}$ & $\begin{array}{l}\text { Control } \\
\text { group }\end{array}$ & P-value & $\begin{array}{l}\text { Experiment } \\
\text { group }\end{array}$ & $\begin{array}{l}\text { Control } \\
\text { group }\end{array}$ & P-value \\
\hline Upper abdominal pain (n) & 50 & 50 & $>0.05^{\mathrm{a}}$ & 4 & 47 & $<0.05^{\mathrm{a}}$ \\
\hline Findings of ultrasonography (n) & 50 & 50 & $>0.05^{\mathrm{a}}$ & 10 & 48 & $<0.05^{\mathrm{a}}$ \\
\hline $\begin{array}{l}\text { Sudden and sharp cutting pains } \\
\text { in upper abdomen }(n)\end{array}$ & 50 & 50 & $>0.05^{\mathrm{a}}$ & 8 & 47 & $<0.05^{\mathrm{a}}$ \\
\hline Abdominal distension (n) & 50 & 50 & $>0.05^{\mathrm{a}}$ & 6 & 49 & $<0.05^{\mathrm{a}}$ \\
\hline Dry and bitter taste (n) & 50 & 50 & $>0.05^{\mathrm{a}}$ & 3 & 46 & $<0.05^{\mathrm{a}}$ \\
\hline $\begin{array}{l}\text { Coated and dry tongue } \\
\text { or burned black tongue (n) }\end{array}$ & 50 & 50 & $>0.05^{\mathrm{a}}$ & 5 & 48 & $<0.05^{\mathrm{a}}$ \\
\hline Forceful sphygmus (n) & 50 & 50 & $>0.05^{\mathrm{a}}$ & 5 & 47 & $<0.05^{\mathrm{a}}$ \\
\hline $\begin{array}{l}\text { Diffuse enlargement of the } \\
\text { pancreas (n) }\end{array}$ & 50 & 50 & $>0.05^{\mathrm{a}}$ & 8 & 48 & $<0.05^{\mathrm{a}}$ \\
\hline White blood cells (cells/ml) & $\begin{array}{l}1.51 \times 10^{5} \\
\pm 42 \times 10^{4}\end{array}$ & $\begin{array}{l}1.53 \times 10^{5} \\
\pm 41 \times 10^{4}\end{array}$ & $>0.05^{\mathrm{b}}$ & $\begin{array}{c}8.51 \times 10^{4} \\
\pm 3.58 \times 10^{4}\end{array}$ & $\begin{array}{l}1.50 \times 10^{5} \\
\pm 40 \times 10^{4}\end{array}$ & $<0.05^{\mathrm{b}}$ \\
\hline Neutrophils (\%) & $68.2 \pm 5.2$ & $68.8 \pm 5.5$ & $>0.05^{\mathrm{b}}$ & $55.7 \pm 4.7$ & $67.3 \pm 5.8$ & $<0.05^{\mathrm{b}}$ \\
\hline Lipase (pg/ml) & $326 \pm 76$ & $318 \pm 81$ & $>0.05^{\mathrm{b}}$ & $207 \pm 57$ & $320 \pm 71$ & $<0.05^{\mathrm{b}}$ \\
\hline Serum amylase (IU/l) & $965 \pm 307$ & $952 \pm 297$ & $>0.05^{\mathrm{b}}$ & $709 \pm 286$ & $943 \pm 255$ & $<0.05^{\mathrm{b}}$ \\
\hline Urine amylase (IU/l) & $1876 \pm 325$ & $1866 \pm 298$ & $>0.05^{\mathrm{b}}$ & $1054 \pm 233$ & $1834 \pm 311$ & $<0.05^{\mathrm{b}}$ \\
\hline
\end{tabular}

P-value calculated by the ${ }^{\mathrm{a} t}$-test and ${ }^{\mathrm{b}} \chi^{2}$ test. AP, acute pancreatitis; ITCM, integrated traditional Chinese medicine.

for $1 \mathrm{~h}$, and then incubated with a primary antibody: Mouse anti-human PRSS1 monoclonal antibody (MAB3848; 1:5,000; R\&D Systems China, Shanghai, China) and mouse anti-human SPINK1 monoclonal antibody (70R-5308; 1:2,000; Beijing Dakewei Bio-Technology Co, Ltd., Beijing, China). A secondary antibody (anti-mouse horseradish peroxidase-conjugated; cat. no. 201201; 1:10,000; Shanghai Guoyuan Biotechnology Co., Ltd., Shanghai, China) was added and chemiluminescence detection performed using an kit from GE Healthcare (Piscataway, NJ, USA).

Statistical analysis. The t-test and the $\chi^{2}$ test were used to detect the statistical significance for the variables between the experimental and control groups. Data were analyzed using SPSS 13.0 software (SPSS, Inc., Chicago, IL, USA), and $\mathrm{P}<0.05$ was considered to indicate a statistically significant difference.

\section{Results}

Characteristics of AP patients. A total of 100 AP patients (64 male, $50.8 \pm 4.2$ years; 36 female, $47.5 \pm 7.5$ years) were recruited. Considering the complicated causative factors for increasing the risk of AP, the gender, age, BMI and daily calorie intake of the patients were not contributing factors (Table I; P>0.05). All these factors were excluded between the experimental and control groups during the data collection. The number of cases of each stage of AP were similar between the experimental and control groups (Table I).

Effects of ITCM on AP patients. Effects of ITCM on the health outcomes of AP patients were investigated, as shown in Table I. All AP patients were diagnosed by gastroenterologists. Following eight days of treatment with ITCM, 29 cases (26 mild, two modest and one severe case) exhibited excellent results, 17 cases (11 modest and six severe) showed an effective response and four cases (severe) exhibited inefficient results. In the control group, one patient exhibited excellent results, two cases showed an effective outcome, while all the other cases resulted in an inefficient outcome. The excellent and effective outcomes in the control group were hypothesized to be the result of natural recovery since the onset of AP. The results demonstrated that ITCM showed strong therapeutic results for AP. In addition, all the symptoms of AP in the experimental group patients were improved significantly when compared with those in the control group $(\mathrm{P}<0.05$; Table II). The results suggested that ITCM was able to ameliorate AP; thus, ITCM should be considered as a potential drug candidate for the treatment of AP.

$m R N A$ expression levels of PRSS1 and SPINK1 in AP patients. The qRT-PCR results revealed that the mRNA expression levels of PRSS1 and SPINK1 were lower in the AP patients treated with ITCM than those prior to treatment. The levels of PRSS1 and SPINK1 were lowest in the excellent outcome experimental group patients, moderate in the effective result patients and highest in the control and inefficient result patients (Fig. 1).

Protein expression levels of PRSS1 and SPINK1 in AP patients. Levels of PRSS1 and SPINK1 proteins are known to be closely associated with the development of AP. The protein expression levels of PRSS1 and SPINK1 were at their 
A
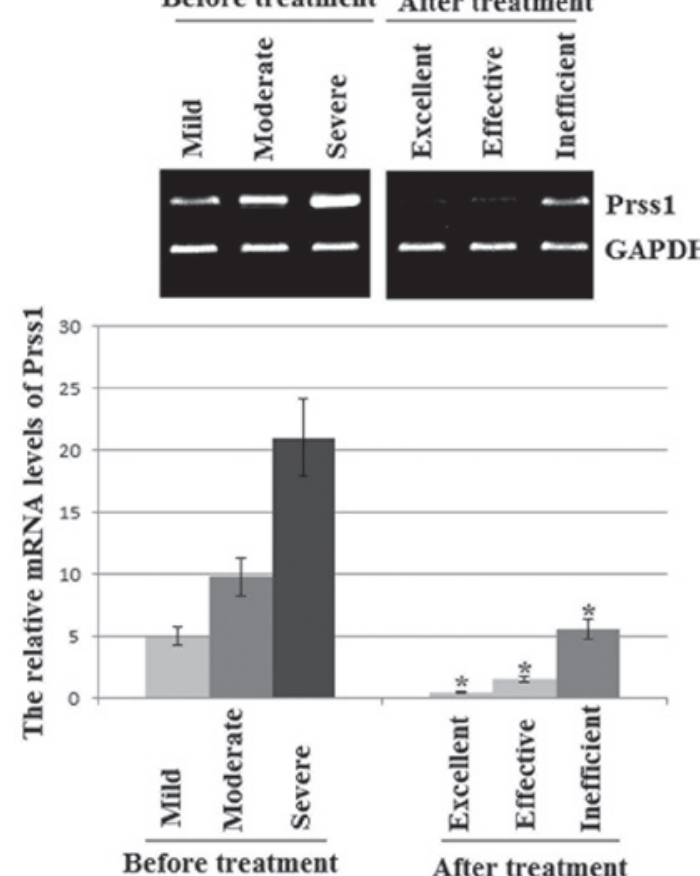

Before treatment

After treatment
B

Before treatment After treatment
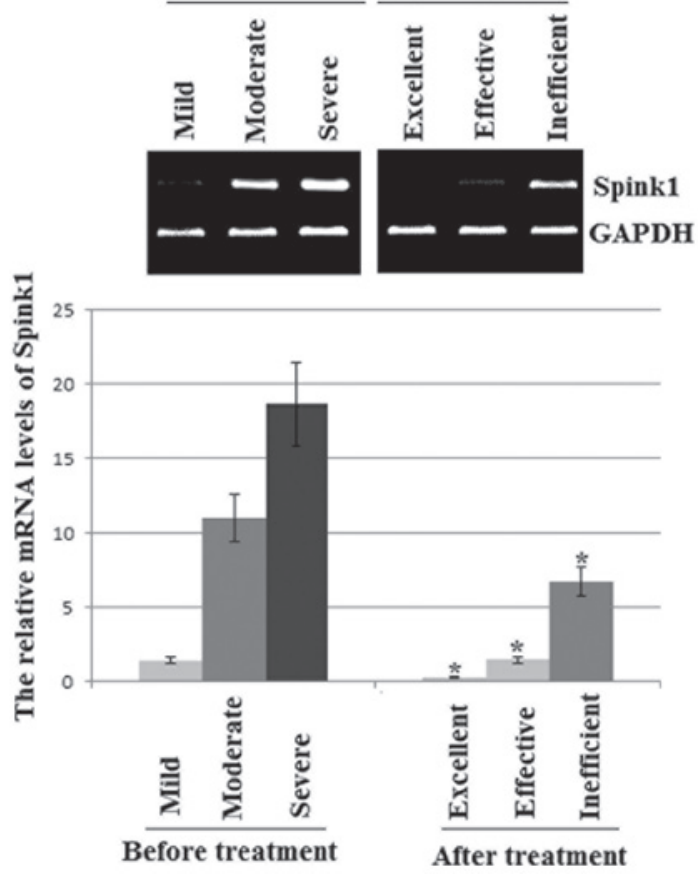

Figure 1. mRNA expression levels of (A) PRSS1 and (B) SPINK1 in AP patients before and after treatment. Patients were divided into three groups based on the severity of their AP; mild, modest and severe. Out of a total of 50 AP patients that underwent ITCM therapy, 26 exhibited mild AP (light grey), 13 exhibited modest AP (medium grey) and 11 patients had severe AP (dark grey). Following ITCM treatment, 29 patients exhibited excellent therapeutic results (26 mild, two modest and one severe), 17 patients exhibited effective results (11 modest and six severe) and four severe patients showed an ineffective response. Data are represented as the mean \pm standard deviation of three independent experiments. " $\mathrm{P}<0.01$, vs. value before treatment. AP, acute pancreatitis; ITCM, integrated traditional Chinese medicine.

A
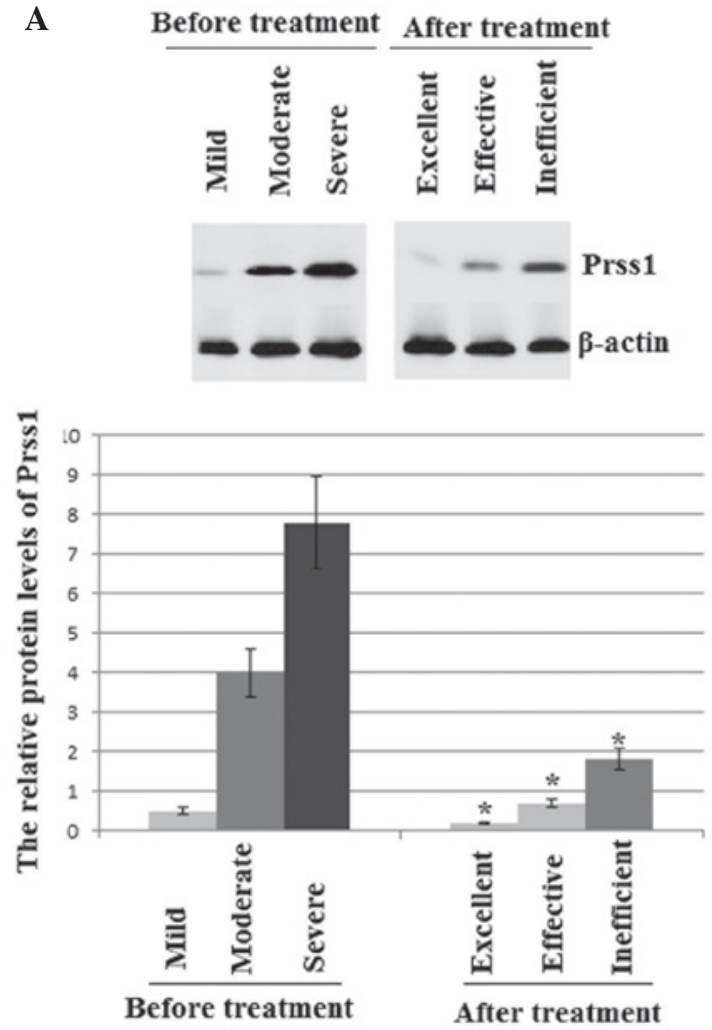

B
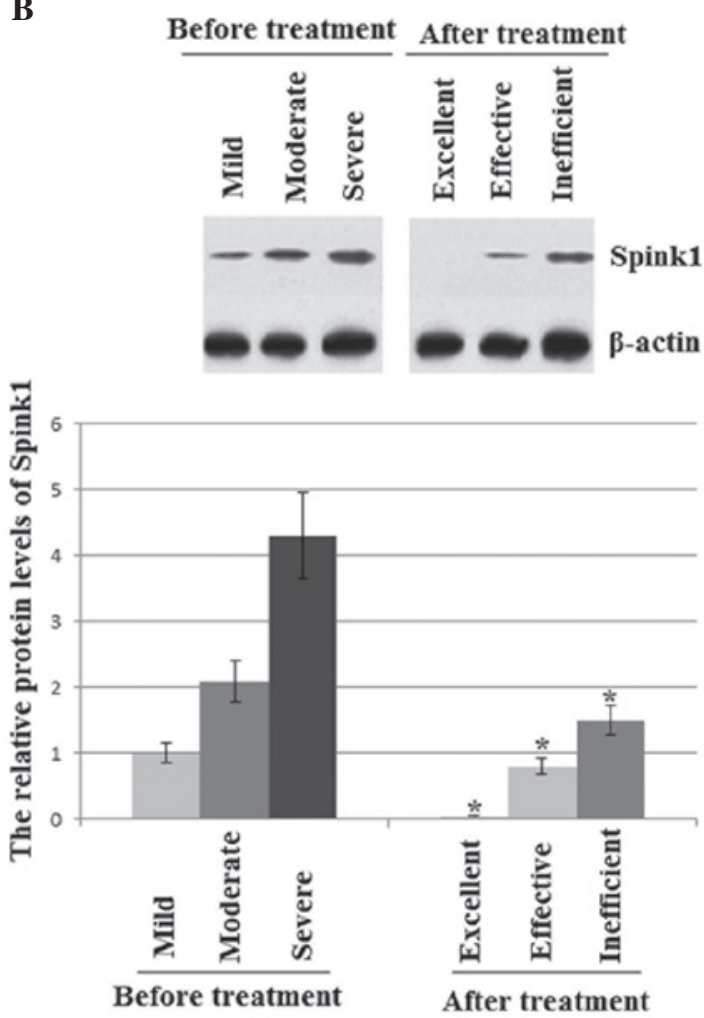

Figure 2. Protein expression levels of (A) PRSS1 and (B) SPINK1 in AP patients before and after treatment. Patients were divided into three groups based on the severity of their AP; mild, modest and severe. Out of a total of 50 AP patients that underwent ITCM therapy, 26 exhibited mild AP (light grey), 13 exhibited modest AP (medium grey) and 11 patients were diagnosed with severe AP (dark grey). Following ITCM treatment, 29 patients exhibited excellent therapeutic results (26 mild, two modest and one severe), 17 patients exhibited an effective outcome (11 modest and six severe) and four severe patients showed an ineffective response. Data are represented as the mean \pm standard deviation of three independent experiments. "P<0.01, vs. value before treatment. AP, acute pancreatitis; ITCM, integrated traditional Chinese medicine. 
highest prior to treatment with ITCM (Fig. 2). Following treatment with ITCM, the protein expression levels of PRSS1 and SPINK1 were lowest in the excellent outcome patients, moderate in the effective outcome patients and highest in the control and inefficient result patients (Fig. 2). Thus, the results were consistent with a previous study (50), which reported that PRSS1 and SPINK1 were potential negative biomarkers for the diagnosis and prognosis of AP.

\section{Discussion}

AP is the most common intestinal disease worldwide, with a mortality rate as high as $36-50 \%$ for severe AP (20). Severe AP can cause serious inflammation in other systems and patients may succumb suddenly to the disease $(14,51)$. Significant progress has been reported in the treatment of AP by ITCM $(21,52,53)$; however, there remain a number of difficulties that hinder the improvement of therapeutic efficacy. TCM methods are commonly used in an integrative manner; thus, the present study aimed to assess the efficacy of an ITCM approach in the therapy of AP.

However, the molecular mechanisms underlying the pathogenesis of AP are not yet known, and a suitable biomarker for AP must be identified in order to further investigate these mechanisms. In certain cases, the levels of a number of important proteins are undetectable in the serum, which may be affected by the instability of human clinical and metabolic conditions. Thus, only newly obtained AP specimens were considered for examination in the present study.

Mutations in the human PRSS1 gene are associated with pancreatitis and have provided insight into the pathogenesis of the disease (54). PRSS1 is widely reported to be associated with AP $(27,55,56)$. In addition, SPINK1 was originally identified as a trypsin inhibitor and is strongly elevated in patients with pancreatitis, where the level of elevation correlates with the disease severity (57). This association between SPINK1 and AP has also been widely reported (24,26,58-63). Thus, the present study aimed to investigate whether the expression levels of PRSS1 and SPINK1 were closely associated with the development of AP.

The sinker-assisted ESD approach to sampling AP patient tissues facilitated the detection of pancreatitis by examination of mRNA levels. The mRNA expression levels were examined initially, following which the protein levels were determined. The mRNA and protein expression levels of PRSS1 and SPINK1 demonstrated the same changed trend with the development of AP (Fig. 1). Therefore, PRSS1 and SPINK1 are potential combined adjuvant biomarkers for investigating the mechanisms underlying the increased risk of AP, and PRSS1 and SPINK1 should be considered as targets for drug therapy. Furthermore, the protein expression levels of PRSS1 and SPINK1 were lower on average in the experimental group when compared with the control group (Fig. 2). Therefore, the development of AP can be characterized by the levels of predominant PRSS1 and SPINK1. Since the expression levels of PRSS1 and SPINK1 were higher in the AP patients of the control group, the changing levels of these biomarkers may be the result of inflammatory processes. The levels of PRSS1 and SPINK1 in the AP patients treated with placebos were comparable to the levels prior to treatment (data not shown).
The application of Chinese herbs to acupuncture points is a type of external TCM. The treatment involves processing the herbs into paste, powder or ointment and subsequently applying them to corresponding acupuncture points $(18,34)$. The interactions among the drugs, main and collateral channels and acupuncture points are involved in the treatment of AP. Medicine is absorbed through the skin, but not the digestive system; thus, the procedure is simple and safe to conduct, with no risk of an adverse reaction. ITCM has been clinically observed to ameliorate a variety of symptoms and conditions, including pain, abdominal distension, constipation, inflammation, endotoxin absorption, enterogenic infection and intestine failure. In addition, ITCM has been shown to improve the levels of amylase in the blood and urine, improve pancreatic blood circulation, promote the absorption of necrotic tissue and gastrointestinal peristalsis and decrease a variety of complications, improving the overall prognosis (64).

A number of important questions should be considered in future studies investigating the effects of ITCM in the treatment of AP. Firstly, the expression levels of PRSS1 and SPINK1 should be assessed in healthy control subjects to better understand the mechanism underlying the therapeutic effects of ITCM. However, it is difficult to recruit appropriate volunteers as the majority of healthy subjects are wary of the potential side effects of surgery based on sinker-assisted ESD techniques. Secondly, the classification system for the various stages of AP requires improvement via the design of a more precise scale.

In conclusion, the present study examined 100 AP patients in order to investigate the effects of ITCM therapy on AP. The results revealed that the development of AP was positively associated with the expression levels of the biomarkers, PRSS1 and SPINK1. Thus, PRSS1 and SPINK1 may be useful combined targets for the treatment of AP. ITCM produced significant therapeutic results for AP when compared with the placebo-treated control group, and should be considered as a potential drug to be developed for the treatment of AP. There are, however, limitations to the present study, and the underlying mechanisms of AP should be investigated further in future research.

\section{References}

1. Matta A, Tandra PK, Cichowski E and Reddymasu SC: Acute necrotising pancreatitis: a late and fatal complication of pancreaticoduodenal arterial embolisation. BMJ Case Rep: bcr2014204197, 2014.

2. Rana SS, Sharma V, Sharma R and Bhasin DK: An unusual complication of acute necrotising pancreatitis detected by endoscopic ultrasound. JOP 15: 276-277, 2014.

3. Ambiru S, Furuyama N, Aono M, et al: Hyperbaric oxygen therapy for the treatment of postoperative paralytic ileus and adhesive intestinal obstruction associated with abdominal surgery: experience with 626 patients. Hepatogastroenterology 54: 1925-1929, 2007.

4. Sheikh I, Fontenot E, Waghray N, et al: The role of nonsteroidal anti-inflammatory drugs in the prevention of post endoscopic retrograde cholangiopancreatography pancreatitis. JOP 15: 219-224, 2014.

5. Sit M, Aktas G, Yilmaz EE, et al: Effects of the inflammatory response on serum omentin levels in early acute and chronic pancreatitis. Clin Ter 165: e148-e152, 2014.

6. Shalimov SA, Popov ON, Dubitskiı̌ AE and Lifshits IuZ: Direct thermometry of the pancreas in the postoperative period. Vestn Khir Im I I Grek 132: 36-39, 1984 (In Russian). 
7. Mascolo N, Izzo AA, Ligresti A, et al: The endocannabinoid system and the molecular basis of paralytic ileus in mice. FASEB J 16: 1973-1975, 2002.

8. Elder AS, Saccone GT, Bersten AD and Dixon DL: Evaluation of lung injury and respiratory mechanics in a rat model of acute pancreatitis complicated with endotoxin. Pancreatology 12 : 240-247, 2012

9. Fujita T: Endotoxin as a trigger of alcoholic pancreatitis. Gastroenterology 134: 640-641, 2008.

10. Verma R, Dhamija R, Ross SC, Batts DH and Loehrke ME: Symbiotic bacteria induced necrotizing pancreatitis. JOP 11 : 474-476, 2010

11. Zhang X, Tian H, Wu C, et al: Effect of baicalin on inflammatory mediator levels and microcirculation disturbance in rats with severe acute pancreatitis. Pancreas 38: 732-738, 2009.

12. Cuthbertson $\mathrm{CM}$ and Christophi $\mathrm{C}$ : Disturbances of the microcirculation in acute pancreatitis. Br J Surg 93: 518-530, 2006.

13. Yang XN, Guo J, Lin ZQ, et al: The study on causes of death in fulminant pancreatitis at early stage and late stage. Sichuan Da Xue Xue Bao Yi Xue Ban 42: 686-690, 2011 (In Chinese).

14. Yuan Z, Meyerholz DK, Twait EC, et al: Systemic inflammation with multiorgan dysfunction is the cause of death in murine ligation-induced acute pancreatitis. J Gastrointest Surg 15: $1670-1678,2011$

15. Tsuji Y, Takahashi N, Fletcher JG, et al: Subtraction color map of contrast-enhanced and unenhanced CT for the prediction of pancreatic necrosis in early stage of acute pancreatitis. AJR Am J Roentgenol 202: W349-W356, 2014.

16. Garip G, Sarandöl E and Kaya E: Effects of disease severity and necrosis on pancreatic dysfunction after acute pancreatitis World J Gastroenterol 19: 8065-8070, 2013.

17. Popa D: Treatment in severe acute pancreatitis - still a reason of debate. J Med Life 6: 486-490, 2013.

18. Ge HY and Chen B: Clinical observation of acute pancreatitis treated with acupoint application combined with medicine. Zhongguo Zhen Jiu 32: 602-604, 2012 (In Chinese).

19. Bossi A, Romeo G and Pezzoli A: Side-effects, structure, and H2-receptor antagonists. Lancet 339: 1366, 1992.

20. Xia Q and Deng LH: Hot issues on the treatment of severe acute pancreatitis by integrated traditional Chinese and Western medicine. Sichuan Da Xue Xue Bao Yi Xue Ban 44: 962-965, 2013 (In Chinese).

21. Wan MH, Yao J, Li J, et al: The effectiveness of purgation and electroacupuncture in extrahepatic bile duct stone complicated with acute biliary pancreatitis: management of biliary stone pancreatitis through traditional Chinese medicine. Pancreas 40 483-484, 2011

22. Tang Y, Liao Y, Kawaguchi-Sakita N, et al: Sinisan, a traditional Chinese medicine, attenuates experimental chronic pancreatitis induced by trinitrobenzene sulfonic acid in rats. J Hepatobiliary Pancreat Sci 18: 551-558, 2011.

23. Solomon S, Whitcomb DC and LaRusch J: PRSS1-related hereditary pancreatitis. In: GeneReviews ${ }^{\circledR}$. Pagon RA, Adam MP, Ardinger $\mathrm{HH}$, et al (eds). University of Washington, Seattle, WA, 2012.

24. Sánchez-Ramírez CA， Flores-Martínez SE García-Zapién AG, et al: Screening of R122H and N29I mutations in the PRSS1 gene and N34S mutation in the SPINK1 gene in Mexican pediatric patients with acute and recurrent pancreatitis Pancreas 41: 707-711, 2012

25. Tukiainen E, Kylänpää ML, Kemppainen E, et al: Pancreatic secretory trypsin inhibitor (SPINK1) gene mutations in patients with acute pancreatitis. Pancreas 30: 239-242, 2005.

26. Lee YJ, Kim KM, Choi JH, et al: High incidence of PRSS1 and SPINK1 mutations in Korean children with acute recurrent and chronic pancreatitis. J Pediatr Gastroenterol Nutr 52: 478-481, 2011.

27. Sobczyńska-Tomaszewska A, Bak D, Oralewska B, et al: Analysis of CFTR, SPINK1, PRSS1 and AAT mutations in children with acute or chronic pancreatitis. J Pediatr Gastroenterol Nutr 43 299-306, 2006

28. Kiriyama S, Gabata T, Takada T, et al: New diagnostic criteria of acute pancreatitis. J Hepatobiliary Pancreat Sci 17: 24-36, 2010.

29. Gubergrits N, Golubova O, Lukashevich G and Fomenko P: Elevated serum amylase in patients with chronic pancreatitis: acute attack or macroamylasemia? Pancreatology 14: 114-116, 2014.

30. Wojtun S and Gil J: Utility of amylase serum and urine activity in acute biliary pancreatitis treated with biliary sphincterotomy. Pol Merkur Lekarski 22: 381-384, 2007 (In Polish).
31. Banks PA, Bollen TL, Dervenis C, et al; Acute Pancreatitis Classification Working Group: Classification of acute pancreatitis - 2012: revision of the Atlanta classification and definitions by international consensus. Gut 62: 102-111, 2013.

32. Li YC, Dong L, Jia A, Chang XM and Xue H: Preparation of solid lipid nanoparticles loaded with traditional Chinese medicine by high-pressure homogenization. Nan Fang Yi Ke Da Xue Xue Bao 26: 541-544, 2006 (In Chinese).

33. Zhuang WJ and Qian C: Treatment of infected deciduous root canal with Samyan (traditional Chinese medicine) paste: Clinical analysis. Shanghai Kou Qiang Yi Xue 6: 185, 1997 (In Chinese)

34. Xue QM, Li N, Xue P, Wang CW and Wen Q: Therapeutic effects of electroacupuncture at ST36 acupoint on sodium-taurocholate-induced severe acute pancreatitis. Chin J Integr Med 20: 695-700, 2014

35. Saito Y, Emura F, Matsuda T, et al: A new sinker-assisted endoscopic submucosal dissection for colorectal cancer. Gastrointest Endosc 62: 297-301, 2005.

36. Zhao J, Liao Q, Zhao Y and Hu Y: Mortality indicators and risk factors for intra-abdominal hypertension in severe acute pancreatitis. Int Surg 99: 252-257, 2014.

37. Vipperla K, Papachristou GI, Easler J, et al: Risk of and factors associated with readmission after a sentinel attack of acute pancreatitis. Clin Gastroenterol Hepatol: May 9, 2014 (Epub ahead of print).

38. Zeng YB, Zhan XB, Guo XR, et al: Risk factors for pancreatic infection in patients with severe acute pancreatitis: an analysis of 163 cases. J Dig Dis 15: 377-385, 2014.

39. Jin J, Yu YH, Zhong M and Zhang GW: Analyzing and identifying risk factors for acute pancreatitis with different etiologies in pregnancy. J Matern Fetal Neonatal Med: June 5, 1-5, 2014 (Epub ahead of print).

40. Tamasaki A, Nishimura Y, Kondo N, et al: Risk factors for acute pancreatitis in patients with severe motor and intellectual disabilities. Pediatr Int 56: 240-243, 2014.

41. Hong S, Qiwen B, Ying J, Wei A and Chaoyang T: Body mass index and the risk and prognosis of acute pancreatitis: a meta-analysis. Eur J Gastroenterol Hepatol 23: 1136-1143, 2011.

42. Shen HN, Wang WC, Lu CL and Li CY: Effects of gender on severity, management and outcome in acute biliary pancreatitis. PLoS One 8: e57504, 2013

43. Fu P, Yuan AH, Wang CH, Li X and Wu HY: Alcohol-induced severe acute pancreatitis followed by hemolytic uremic syndrome managed with continuous renal replacement therapy. BMC Nephrol 15: 1, 2014.

44. Nikkola J, Räty S, Laukkarinen J, et al: Abstinence after first acute alcohol-associated pancreatitis protects against recurrent pancreatitis and minimizes the risk of pancreatic dysfunction. Alcohol Alcohol 48: 483-486, 2013.

45. Müller S, Kaiser H, Krüger B, et al: Age-dependent effects of UCP2 deficiency on experimental acute pancreatitis in mice. PLoS One 9: e94494, 2014.

46. Okamura D, Starr ME, Lee EY, et al: Age-dependent vulnerability to experimental acute pancreatitis is associated with increased systemic inflammation and thrombosis. Aging Cell 11: 760-769, 2012.

47. Akesson A, Andersen LF, Kristjánsdóttir AG, et al: Health effects associated with foods characteristic of the Nordic diet: a systematic literature review. Food Nutr Res: 57, 2013.

48. Overby NC, Sonestedt E, Laaksonen DE and Birgisdottir BE: Dietary fiber and the glycemic index: a background paper for the Nordic Nutrition Recommendations 2012. Food Nutr Res: 57, 2013.

49. Knaan T, Endevelt R and Singer P: Evaluating the validity of the 'categories method': A new method for self-report assessment of daily calorie intake: A pilot study. Can J Diabetes 37 (Suppl 2): S283, 2013.

50. Pelaez-Luna M, Robles-Diaz G, Canizales-Quinteros S and Tusie-Luna MT: PRSS1 and SPINK1 mutations in idiopathic chronic and recurrent acute pancreatitis. World J Gastroenterol 20: 11788-11792, 2014

51. Roncati L, Gualandri G, Fortuni G and Barbolini G: Sudden death and lipomatous infiltration of the heart involved by fat necrosis resulting from acute pancreatitis. Forensic Sci Int 217: e19-e22, 2012.

52. Chen H,Li F, Jia JG, et al: Effects of traditional Chinese medicine on intestinal mucosal permeability in early phase of severe acute pancreatitis. Chin Med J (Engl) 123: 1537-1542, 2010. 
53. Zhang MJ, Zhang GL, Yuan WB, Ni J and Huang LF: Treatment of abdominal compartment syndrome in severe acute pancreatitis patients with traditional Chinese medicine. World J Gastroenterol 14: 3574-3578, 2008.

54. Athwal T, Huang W, Mukherjee R, et al: Expression of human cationic trypsinogen (PRSS1) in murine acinar cells promotes pancreatitis and apoptotic cell death. Cell Death Dis 5: e1165, 2014.

55. Wang J, Ohmuraya M, Suyama K, et al: Relationship of strain-dependent susceptibility to experimentally induced acute pancreatitis with regulation of Prss1 and Spink3 expression. Lab Invest 90: 654-664, 2010.

56. Corleto VD, Gambardella S, Gullotta F, et al: New PRSS1 and common CFTR mutations in a child with acute recurrent pancreatitis, could be considered an 'Hereditary' form of pancreatitis? BMC Gastroenterol 10: 119, 2010.

57. Ohmuraya M and Yamamura K: Roles of serine protease inhibitor Kazal type 1 (SPINK1) in pancreatic diseases. Exp Anim 60: 433-444, 2011.

58. Rai P, Sharma A, Gupta A and Aggarwal R: Frequency of SPINK1 N34S mutation in acute and recurrent acute pancreatitis. J Hepatobiliary Pancreat Sci 21: 663-668, 2014.
59. Tremblay K, Dubois-Bouchard C, Brisson D and Gaudet D: Association of CTRC and SPINK1 gene variants with recurrent hospitalizations for pancreatitis or acute abdominal pain in lipoprotein lipase deficiency. Front Genet 5: 90, 2014.

60. Terlizzi V, De Gregorio F, Sepe A, et al: Brand new SPINK1 and CFTR mutations in a child with acute recurrent pancreatitis: a case report. Minerva Pediatr 65: 669-672, 2013.

61. Jøergensen MT, Brusgaard K, Novovic S, et al: Is the SPINK1 variant p.N34S overrepresented in patients with acute pancreatitis? Eur J Gastroenterol Hepatol 24: 309-315, 2012.

62. Baudry C, Rebours V, Houillier P, et al: Recurrent acute pancreatitis caused by association of a novel mutation of the calcium-sensing receptor gene and a heterozygous mutation of the SPINK1 gene. Pancreas 39: 420-421, 2010.

63. Aoun E, Muddana V, Papachristou GI and Whitcomb DC: SPINK1 N34S is strongly associated with recurrent acute pancreatitis but is not a risk factor for the first or sentinel acute pancreatitis event. Am J Gastroenterol 105: 446-451, 2010.

64. Liu XB, Jiang JM, Huang ZW, et al: Clinical study on the treatment of severe acute pancreatitis by integrated traditional Chinese medicine and Western medicine. J Sichuan University (medical science edition) 35: 204-208, 2004 (In Chinese). 